\title{
28 Research Square \\ Analysis Results of COVID-19 Data by GIS at Different Scales
}

yanxiang li

School of Surveying and Land Information Engineering, Henan Polytechnic University, 454000, China, Jiaozuo

zhanliang yuan ( $\square$ yuan6400@hpu.edu.cn )

School of Surveying and Land Information Engineering, Henan Polytechnic University, 454000, China, Jiaozuo https://orcid.org/0000-0001-8124-3263

qiaoqiao wang

Institute of Resources \& Environment, Henan Polytechnic University, 454000, China, Jiaozuo

\section{Research article}

Keywords: COVID-19, GIS, Multi-scale visualization, Spatial analysis

Posted Date: August 7th, 2020

DOI: https://doi.org/10.21203/rs.3.rs-50633/v1

License: (1) (1) This work is licensed under a Creative Commons Attribution 4.0 International License. Read Full License 


\section{Abstract}

Since the emergence of the new corona virus Corona Virus Disease 19 (COVID-19), it has spread rapidly around the world and has had a serious impact on world economic and social development. In this article, we reviewed the pathogenesis, development process, and the impact and loss of COVID-19 on global human health. Combined with the GIS method, the global distribution of COVID-19 was visualized at multiple scales, and the core areas of the COVID-19 epidemic at different scales were determined. And use the global real-time updated infection data to judge the overall development trend of COVID-19. After visualizing and analyzing the global epidemic data, it was found that the number of patients with COVID19 at the global scale is still growing at a relatively rapid rate, but the global average mortality rate is drop rapidly. The country with the highest mortality rate at the national scale is $16.24 \%$ in Belgium, followed by France and Italy, with mortality rates of $15.31 \%$ and $14.4 \%$, respectively. Changes in the number of COVID19 cases in the United States at the regional scale have a major impact on the global epidemic. Although the mortality rate in the United States is gradually declining, the number of infections and deaths is still increasing at a relatively rapid rate. At the regional scale, the New York epidemic is a hot spot in the United States and should be given high priority.

\section{Introduction}

In December 2019, several cases of pneumonia of unknown etiology were found in the South China Seafood Wholesale Market in Wuhan City, Hubei Province, China ${ }^{1-3}$. On December 31, 2019, China notified the World Health Organization of the epidemic situation. January 26, 2020 has caused 2,794 laboratory confirmed infections, including 80 deaths $^{4}$. On February 11, 2020, the International Virus Taxonomy Commission called this virus Severe Acute Respiratory Syndrome Coronavirus 2 (SARS-CoV$2)^{5}$. SARS-CoV-2 is an animal virus belonging to the genus Coronavirus ${ }^{6}$. This is an RNA virus. Because it has a diameter of 60 to 140 nanometers, there are spikes on the surface, and it is crowned under the electron microscope. Virus hosts may be bats, snakes, seafood and pangolins ${ }^{7,8}$. According to the results of the current genome-wide sequencing analysis, SARS-CoV- 2 is $96 \%$ homologous to the bat coronavirus, so it is judged that the virus uses bat as the original host ${ }^{4}$, but the intermediate host is not yet known ${ }^{9}$. Since the virus first appeared in Wuhan, it has spread rapidly in other provinces, cities, and other countries in China ${ }^{10}$, causing great public health concerns worldwide ${ }^{11}$. On January 30,2020 , the World Health Organization declared the 2019 new coronary pneumonia epidemic as "an emergencies public health incident of international concern"12. On February 11, 2020, the Director General of the World Health Organization Tan Desai announced in Geneva that the pneumonia infected by the new coronavirus was named "COVID-19"13 . Since the outbreak of COVID-19, the number of cases has shown a rapid upward trend. Analysis of relevant data shows that there have been cases that have not been to the South China Seafood Market in Wuhan or have been exposed to live poultry, and some cases have not traveled to China. These phenomena indicate that these cases People-to-people phenomena have occurred locally in the country or region where they are located ${ }^{14}$. When a patient coughs or sneezes, a large amount of infected large droplets are generated ${ }^{14}$, which can be spread to others. The human-to-human 
phenomenon means that COVID-19 patients and asymptomatic patients become the main source of infection $^{2,15}$. The clinical features of COVID-19 are diverse, ranging from asymptomatic to acute respiratory distress syndrome and multiple organ dysfunction or even death ${ }^{15-17}$. As of June 5,2020 , a total of 188 countries or regions have reported more than 6.7 million confirmed cases to the World Health Organization, of which more than 390,000 patients died ${ }^{18}$. After the infection and outbreak of COVID-19 in the world, researchers in different fields have conducted a lot of research on COVID-19 from their respective fields. As early as February, Chinese researchers used spatial analysis methods to show the geographical distribution and changes of the epidemic ${ }^{11,19}$; the study also investigated the three variable biology of COVID-19 coronavirus infection prevalence and mortality outbreak And epidemiological trends ${ }^{20}$; selective pressure analysis of the virus was carried out through modeling, and the transmembrane spiral segments of viruses nsp2 and nsp3 were detected ${ }^{21,22}$; some drugs intended for treatment were found to be arbidol (an antiviral drug from Russia and China) ), intravenous injection of immunoglobulin, interferon, chloroquine and COVID-19 plasma of rehabilitation patients. Various countries are actively studying therapeutic drugs and vaccines, and studies have found that remdesivir, lopinavir and ritonavir may be effective for COVID-1923. The COVID-19 vaccine developed by Zhu F-C and others has entered the first phase of clinical trials and released the human clinical data of the first new crown vaccine to the world ${ }^{24}$. France, Germany, Italy and the Netherlands have formed a "vaccine alliance" to accelerate the development of vaccines in Europe to combat the new coronavirus ${ }^{25}$.

These studies have provided guidelines for the global fight against viruses. With the further spread of the epidemic, it has had a non-negligible impact on the global social, economic, medical system, and even the environment. The 2019 Coronavirus (COVID-19) global pandemic not only caused infection and death, but also caused the worst damage to the global economy since the Great Depression. Since the outbreak of the epidemic, various countries have taken different measures to minimize trade exchanges and reduce the flow of people, resulting in a rapid decline in world economic trade. COVID-19 has the potential to destroy personal livelihoods, businesses, industry and the entire economy ${ }^{26}$.In order to jointly deal with the virus crisis, people are also actively responding. In addition to the policies and regulations issued by local governments and appeals to people to minimize outings and reduce crowding, the World Health Organization has also issued a global strategy to deal with the COVID-19 pandemic ${ }^{27}$.

\section{Data And Methods}

The data used in this article comes from the Github open source projects CovidData and The New York Times. The CovidData project data is COVID-19 daily data. From January 22, 2020 to the present, it contains three different spatial scales of infection, death and recovery cases in countries, cities and regions ${ }^{28}$. The covid-19-data project in The New York Times provides infections and deaths in various regions of the United States ${ }^{29}$. Geographic information technology can reveal the spatial and temporal pattern and spatial distribution of the epidemic in epidemic prevention and control, and display the status of the epidemic at different scales, which can give people a more intuitive feeling. In this study, the spatial 
analysis method will be used to analyze the global epidemic data based on ArcGIS 10.7, so that people can have a deeper understanding of the global status of COVID-19.

\section{Multiscale Analysis}

\subsection{COVID-19 Global Data Analysis}

As of June 5, 2020, there were more than 6.7 million confirmed cases of COVID-19 in 188 countries and regions around the world, and a total of more than 390,000 deaths. The picture of Case Comparison (Fig.1) shows that the Americas and Europe are the regions with the largest number of infections ${ }^{30}$. According to statistics, there are 3748633 cases of COVID-19 ending in the world, of which 3350389 cases accounted for $89 \%$, and 398244 deaths accounted for $11 \% ; 3100946$ cases of existing infections, including 3047330 cases of mild patients accounted for $98 \%$, severe patients 53616 cases accounted for $2 \%^{31}$.

The analysis of the cumulative confirmed cases from January 22, 2020 to June 05,2020 found that the global spread of COVID-19 is divided into three stages: the virus spreads at a relatively rapid rate during the initial outbreak (January to February), and grows faster (Fig.2 A); in the end of March, the cumulative number of global COVID-19 cases increased exponentially, and COVID-19 entered the global outbreak period (Fig.2 B); since early April, the cumulative number of global cases has risen linearly for a long time Maintaining this trend, as of June 05,2020 , the number of newly diagnosed COVID-19s per day has exceeded 100,000 (Fig.2 C).

The rapid spread of COVID-19 around the world has caused more and more people to die from the virus. Since the outbreak, at least 390,000 people worldwide have died from the virus. Global statistics on deaths have found that the global cumulative death toll curve of COVID-19 (Fig.3) is similar to the global number of diagnosed patients (Fig.2), The COVID-19 global cumulative number of deaths curve has a strong similarity with the global trend of diagnosed people over time (e.g. Fig.2 A, B. Fig.3 D, E, F), indicating that there is a positive correlation between the number of diagnoses and the number of deaths. Since the global prevalence of COVID-19, the lowest point of the global average mortality rate is $2.04 \%$ and the highest point is $7.17 \%$. The overall change trend of the global average mortality rate is divided into three stages: the initial stage of the outbreak (2020/01/22-2020/02/05) produces the lowest point from high to low; after the global outbreak (2020/02/06-2020/05/15) The gradual increase in mortality yields the highest point; after the global pandemic (as of now (2020/05/16-2020/06/05), the mortality rate has gradually declined. The important turning point information of the global mortality change is shown in the figure (Fig.3 R1, R2, R3).

Through the analysis of global COVID-19 data, we can see that the spread of the epidemic is divided into three stages: the initial outbreak period, the outbreak period, and the global pandemic period. The latest data shows that COVID-19 is still on the rise in the world, and the number of infected people is increasing, but the mortality rate has been effectively controlled. 


\subsection{COVID-19 Visual analysis of national data}

Visual analysis of COVID-19 data from different countries can further refine the global epidemic distribution. In this study, ArcGIS 10.7 was used to visualize the latest national infection data, and the spatial distribution of the global epidemic situation was visually indicated by a hierarchical color display \Fig.4】. In Fig. 4, different countries are classified according to the number of COVID-19 cases, which are divided into 10 levels. It can be seen from the figure that the spread of COVID-19 has caused infection in most countries in the world, and the infection in the Americas is the most serious.

Through data screening, there are 14 countries with more than 100,000 COVID-19 infections in the world. After sorting, we get Fig. 5. The three countries with the largest number of infections in the world are United States, Brazil and Russia. The time series curves of the number of infected people in 14 countries are drawn in Fig. 6. Among them, the case growth rate in the United States is the fastest, and it has maintained rapid growth for a long time. Therefore, the current situation of the US COVID-19 epidemic is very worrying. In addition, the number of infected people in Brazil and Russia also showed a strong growth trend.

The severe epidemic situation in the world has forced us to understand the situation in various countries. This paper visually analyzes the mortality rates of different countries around the world, and obtains the Fig.7 global mortality distribution map, and screens out the top 10 countries for global mortality (such as Fig.8). It can be seen from Fig.7 and Fig.8 that the countries with the highest mortality rates in the world are mainly distributed in the western coastal countries of North America and Europe. The countries with the highest mortality rate are Belgium with a high of $16.24 \%$, followed by France and Italy with a mortality rate of $15.31 \%$ and $14.4 \%$.

\subsection{Analysis of the current situation in the US}

Through global data analysis and national data visualization analysis, it is found that the global case time series curve (Fig.2) and the US case number time series curve (Fig.6) have strict similarity and the time change nodes of the two curves are very consistent. Therefore, we can determine that changes in the US epidemic affect the direction of the global epidemic. As of June 05,2020 , the number of diagnoses in the United States has reached $1,897,380$, accounting for $28.17 \%$ of the total number of global infections. Therefore, a data report that analyzes the current situation of the US epidemic in detail is particularly important, which makes people have a more intuitive understanding of the current status of the US COVID-19.

\subsubsection{Spatial distribution}

The directional distribution (standard deviation ellipse) can be used to summarize the spatial characteristics of geographic features: central trend, dispersion, and directional trend ${ }^{32}$. In this study, the number of infected people in major areas of the United States was selected as the analysis object, and a 
color map of COVID-19 infection cases was established, and the standard deviation ellipse was established using 1 STANDARD DEVIATION to obtain Fig.9. As can be seen from the color band values in the legend, the number of infected people in each state of the United States is up to 381,019 . The direction distribution (standard deviation ellipse) shows that the US epidemic center is biased toward the eastern United States.

In order to further determine the focus areas of the US epidemic, this study conducted a hot spot analysis (Getis-Ord Gi*) based on Fig.9 and obtained Fig.10. It can be seen from the figure that the hot spot area of the US epidemic is near New York State, with 99\% confidence that the area is the US epidemic center.

Therefore, several areas adjacent to New York should strengthen epidemic management and control, and control hot spots from spreading further.

\subsubsection{The United States data analysis}

Further analysis of the epidemic data in the United States, through time series visualization (Fig.11) of infection cases, deaths and mortality found: 1 . The number of infections and deaths showed the same change trend and had a strong correlation; 2 . The mortality curve fluctuated greatly The highest mortality rate is 7.19 , which is 0.02 higher than the maximum average mortality rate in the world, which is about two months ahead of the maximum global average mortality rate; 3 . At this stage, the COVID-19 mortality rate in the United States is maintaining a downward trend (Fig.11 R), and the downward trend is obvious.

\section{Conclusions}

The global pandemic of COVID-19 has had a significant impact on all countries in the world. Since the outbreak of COVID-19, it has spread to 188 countries and regions, including 14 countries with more than 100,000 cases, and 1.89 million in the United States. In the top ten countries with the lowest mortality rate, the lowest mortality rate is $8.5 \%$, and the highest is $16.24 \%$. The study found that the time series curve of the number of COVID-19 infections in the world and the time series curve of the number of COVID-19 infections in the United States have the same change interval and change trend. The infection cases in the United States account for $28.17 \%$ of the global infections. It can be seen that the change trend of the number of infections in the United States can affect the general direction of change in the global epidemic.

In this study, based on GIS spatial analysis, spatial statistics and time series visualization methods, multiscale visual analysis of global epidemic data was carried out, revealing the multi-scale spatial and temporal pattern and temporal and spatial changes of COVID-19. Multi-scale analysis of COVID-19 can provide support for global epidemic prevention and control and regional epidemic management, so that people have an intuitive understanding of the status of the epidemic. The COVID-19 pandemic poses a huge threat to the lives and health of people around the world. It is the responsibility of all humanity to effectively prevent and control the spread of viruses. It is hoped that the work done in this study will help the global epidemic management. 


\section{Declarations}

- Ethics approval and consent to participate

Not applicable.

- Consent for publication

Not applicable.

- Availability of data and materials

The datasets analysed during the current study are available in the https://github.com/coviddata/coviddata\#csvs.

- Competing interests

The authors declare that they have no competing interests.

- Funding

The authors declare that the study is unfunded.

- Authors' contributions

Li Yanxiang is the main completion person of this paper, responsible for data analysis and data visualization, responsible for drawing and sorting out Figure 2, Figure 3, Figure 6, Figure 10 and Figure 11, and responsible for completing the first draft.

Yuan Zhanliang was responsible for adjusting the research ideas and methods of the article, coordinating and guiding the orderly description of the article, and finally reviewing and finalizing the article.

Wang Qiqiaoqiao was mainly responsible for data collection, drawing and analysis of Figure 1, Figure 4, Figure 5, Figure 7, Figure 8 and Figure 9 in the paper, reviewing and checking the first draft and making modifications.

- Acknowledgements

Thanks to The guidance provided by Dr. Yuan in this research, Dr. Yuan has been engaged in the research of geographic information for a long time and has made great contributions to the proposal of the research route in this paper. I would like to thank Dr. Jin for his constructive comments and discussion on the graphic format of this paper. I would like to thank Master Wang for her great efforts in data collection, collation and introduction description.

\section{References}


1. Li, Q. et al. Early transmission dynamics in Wuhan, China, of novel coronavirus-infected pneumonia. $N$. Engl. J. Med.382, 1199-1207 (2020).

2. Zhu, N. et al. A novel coronavirus from patients with pneumonia in China, 2019. N. Engl. J. Med.382, 727-733 (2020).

3. Wang, D. et al. Clinical Characteristics of 138 Hospitalized Patients with 2019 Novel CoronavirusInfected Pneumonia in Wuhan, China. JAMA - J. Am. Med. Assoc.323, 1061-1069 (2020).

4. Zhou, P. et al. A pneumonia outbreak associated with a new coronavirus of probable bat origin. Nature579, 270-273 (2020).

5. Gorbalenya, A. E. et al. The species and its viruses - a statement of the Coronavirus Study Group. Biorxiv (Cold Spring Harb. Lab. 1-15 (2020) doi:10.1101/2020.02.07.937862.

6. Hernández, J. C. \& Ramzy, A. China Confirms New Coronavirus Spreads From Humans to Humans The New York Times. The New York Times (2020).

7. Ji, W., Wang, W., Zhao, X., Zai, J. \& Li, X. Cross-species transmission of the newly identified coronavirus 2019-nCoV. Journal of Medical Virology vol. 92 (2020).

8. Zhang, T., Wu, Q. \& Zhang, Z. Pangolin homology associated with 2019-nCoV. bioRxiv 2020.02.19.950253 (2020) doi:10.1101/2020.02.19.950253.

9. Xie, P., Ma, W., Tang, H. \& Liu, D. Severe COVID-19: A Review of Recent Progress With a Look Toward the Future. Front. Public Heal.8, 1-7 (2020).

10. Wang, C., Horby, P. W., Hayden, F. G. \& Gao, G. F. A novel coronavirus outbreak of global health concern. Lancet395, 470-473 (2020).

11. Meo, S. A. et al. Biological and epidemiological trends in the prevalence and mortality due to outbreaks of novel coronavirus COVID-19. J. King Saud Univ. - Sci. (2020) doi:10.1016/j.jksus.2020.04.004.

12. WHO. WHO | WHO in emergencies. Who (2020).

13. WHO named novel Coronavirus pneumonia COVID_19.

14. Rothe, C. et al. Transmission of 2019-NCOV infection from an asymptomatic contact in Germany. N. Engl. J. Med.382, 970-971 (2020).

15. Guan, D. Clinical characteristics of 2019 novel coronavirus infection in China. 18, 515-520 (2020).

16. Li, X. et al. Clinical characteristics of 25 death cases with COVID-19: A retrospective review of medical records in a single medical center, Wuhan, China. Int. J. Infect. Dis.94, 128-132 (2020). 
17. Yang, X. et al. Clinical course and outcomes of critically ill patients with SARS-CoV-2 pneumonia in Wuhan, China: a single-centered, retrospective, observational study. Lancet Respir. Med.8, 475-481 (2020).

18. Newsgroup, B. V. and D. Covid-19- a look at the latest global data.

19. Weiming T. , Huipeng L., G. M. The changing patter of COVID-19 in China: A tempo-geographic analysis of the SARS-CoV-2 epidemic. (2020).

20. Angeletti, S. et al. COVID-2019: The role of the nsp2 and nsp3 in its pathogenesis. J. Med. Virol.92, 584-588 (2020).

21. Ying-Hui Jin , Lin Cai , Zhen-Shun Cheng. A rapid advice guideline for the diagnosis and treatment of 2019 novel coronavirus (2019-nCoV) infected pneumonia (standard version). Pediatr. Integr.24, 69-70 (2020).

22. Zhang, L. \& Liu, Y. Potential interventions for novel coronavirus in China: A systematic review. Journal of Medical Virology vol. 92 (2020).

23. Holshue, M. L. et al. First case of 2019 novel coronavirus in the United States. N. Engl. J. Med.382, 929-936 (2020).

24. Zhu, F.-C. et al. Safety, tolerability, and immunogenicity of a recombinant adenovirus type- 5 vectored COVID-19 vaccine: a dose-escalation, open-label, non-randomised, first-in-human trial. Lancet6736, 1-10 (2020).

25. an alliance of four European countries speeds up the development of COVID-19 vaccine.

26. Laing, T. The economic impact of the Coronavirus 2019 (Covid-2019): Implications for the mining industry. Extr. Ind. Soc.2019, (2020).

27. WHO. Strategy and Planning. Enterprise Security Architecture 167-168 (2020) doi:10.1201/b1777611.

28. coviddata_coviddata_Daily COVID-19 statistics by country, region, and city. Github https://github.com/coviddata/coviddata\#csvs (2020).

29. covid-19-data_live at master · nytimes_covid-19-data. https://github.com/nytimes/covid-19-data.

30. World Health Organization. WHO Coronavirus Disease (COVID-19) Dashboard. Covid-19 Dashboard 1-1 https://covid19.who.int/?gclid=CjwKCAjw5lj2BRBdEiwA0Frc9T_Aqdps8|451uvP5utJvHZwFwM65LegPzc_U0uYe2Cs58PZyb2CRoC_FIQAvD_BwE (2020).

31. Worldometer. Coronavirus Update (Live): Cases and Deaths from COVID-19 Virus Pandemic. Worldometers 1 
https://www.worldometers.info/coronavirus/\%0Ahttps://www.worldometers.info/coronavirus/? (2020).

32. Wang, B., Shi, W. \& Miao, Z. Confidence analysis of standard deviational ellipse and its extension into higher dimensional Euclidean space. PLoS One10, 1-17 (2015).

\section{Figures}

\section{Americas}

\section{Europe}

Eastern Mediterranean

South-East Asia

Western Pacific

Africa

I

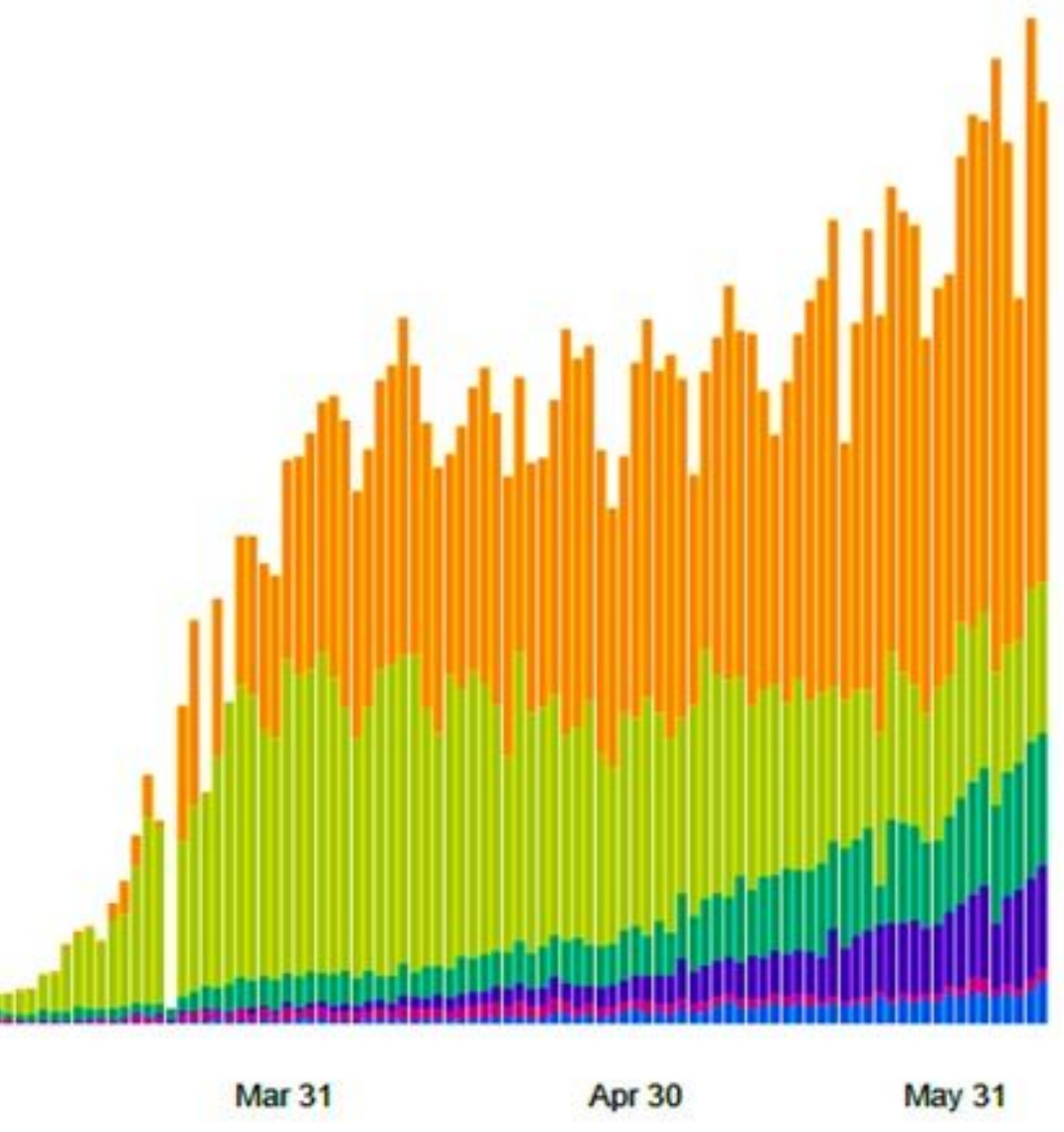

Figure 1

Case Comparison (WHO Regions)Source: World Health Organization 
A

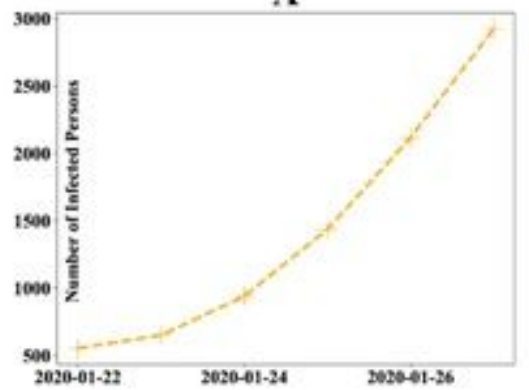

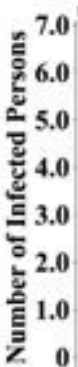

B

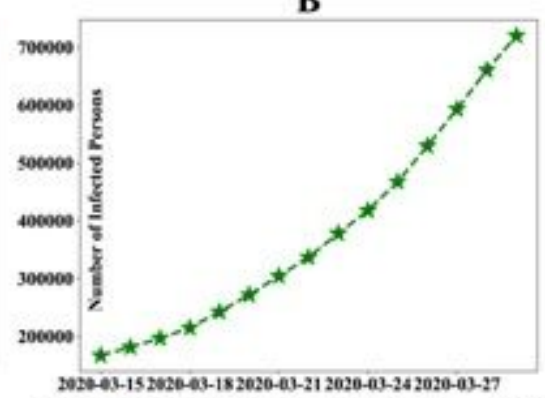

C

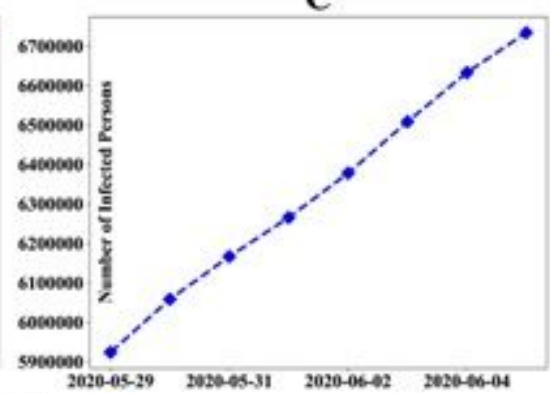

COVID-19 Global Infection Total

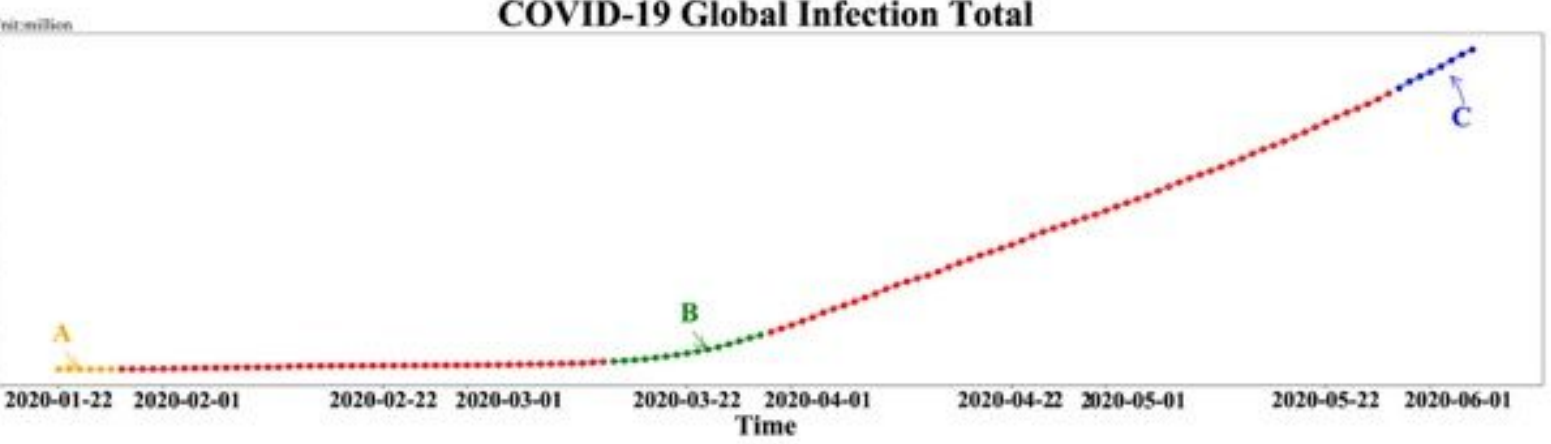

\section{Figure 2}

Globally confirmed number of people. A represents the number of global infections in the initial stage of the virus outbreak; B represents the number of global infections in the stage of rapid virus outbreak; $C$ represents the number of global infections in the latest stage. 
D
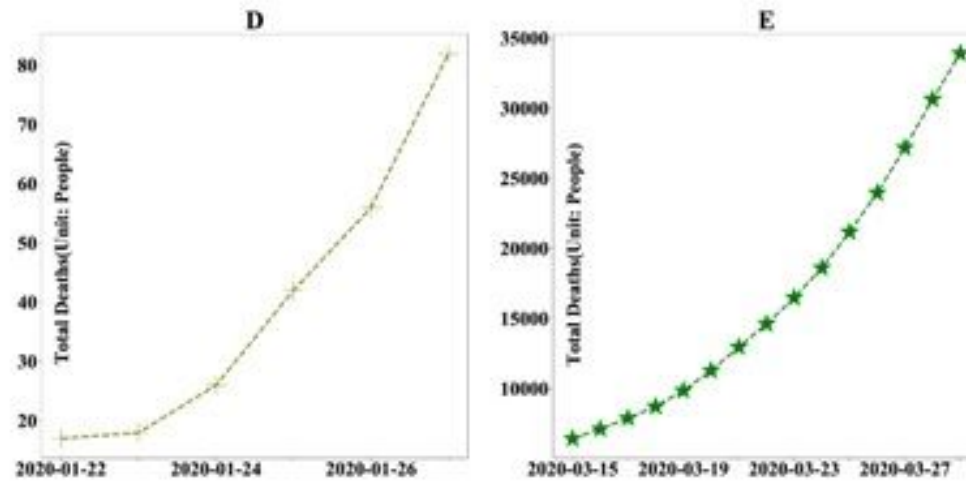

$\mathbf{F}$

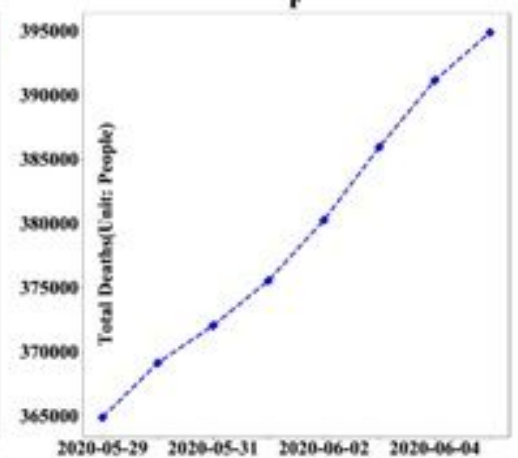

$2020-05-29 \quad 2020-05-31 \quad 2020-06-02 \quad 2020-06-04$

COVID-19 Global Death Toll and Mortality

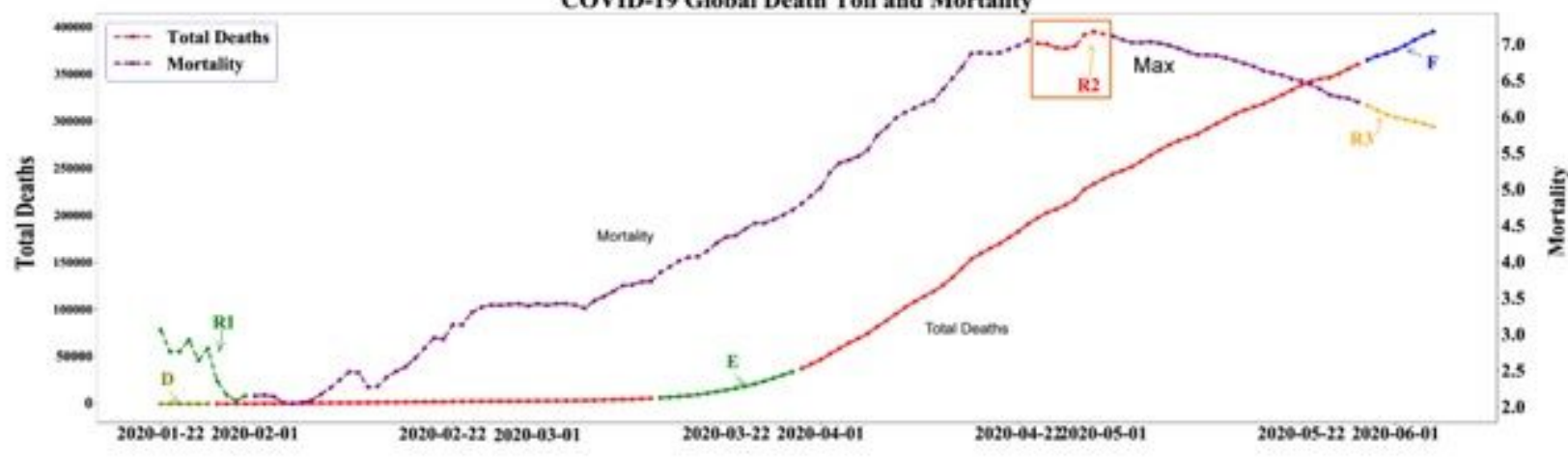

R1

R2
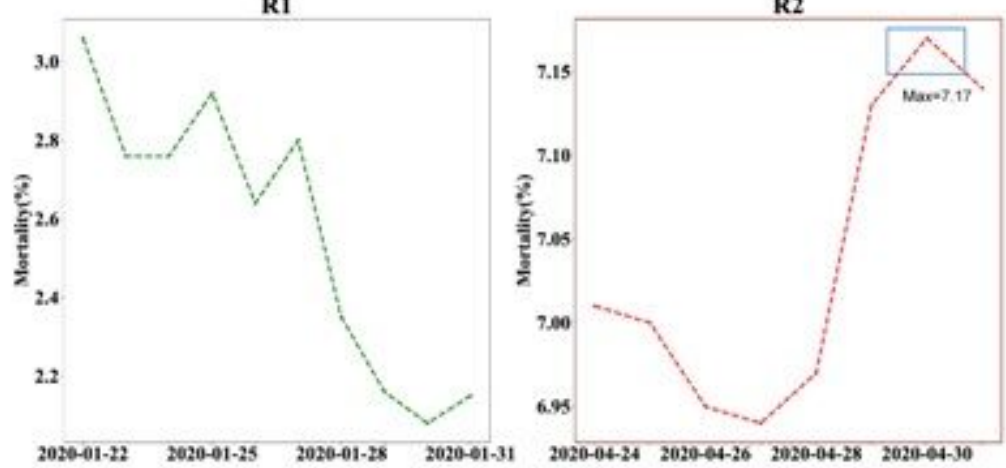

R3

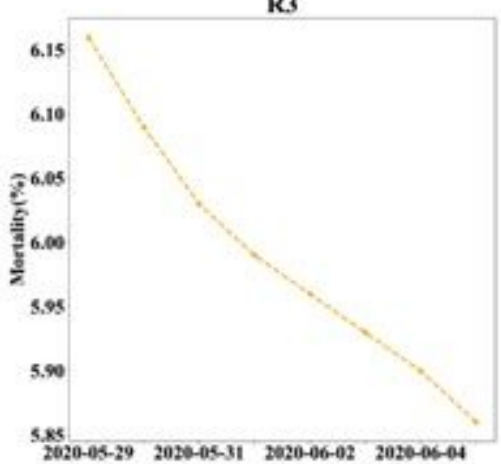

\section{Figure 3}

COVID-19 Global Infection Number and Mortality.D is the number of global deaths at the beginning of the virus outbreak; $E$ is the number of global deaths during the rapid spread of the virus; $F$ represents the global death toll in the latest stage; R1 represents the global average mortality rate at the beginning of the virus outbreak; R2 represents the global average mortality rate during the virus outbreak; R3 represents the global average death rate at the latest stage. 


\section{Distribution of COVID-19 Infected Countries}

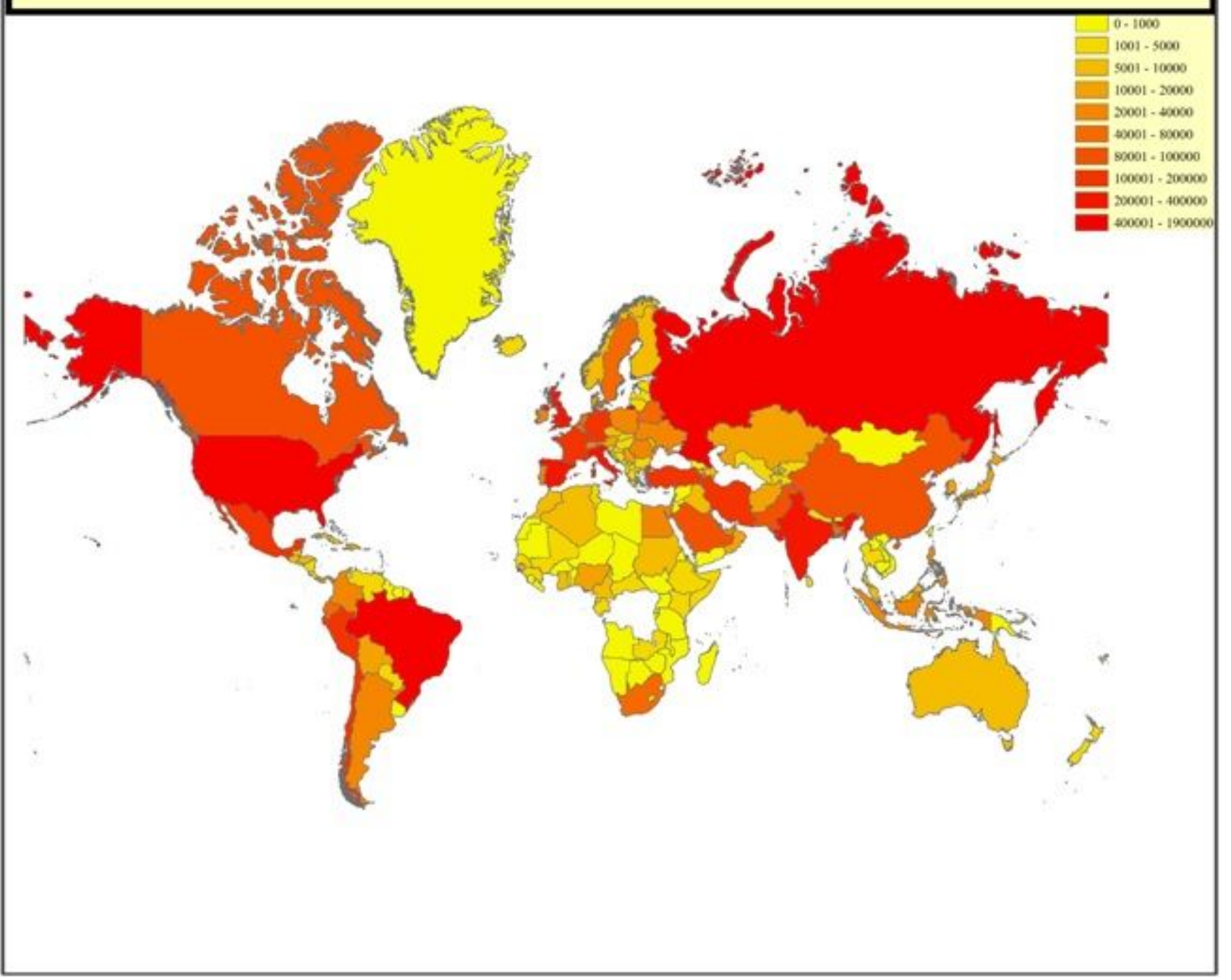

\section{Figure 4}

Distribution of COVID-19 Infected Countries. Note: The designations employed and the presentation of the material on this map do not imply the expression of any opinion whatsoever on the part of Research Square concerning the legal status of any country, territory, city or area or of its authorities, or concerning the delimitation of its frontiers or boundaries. This map has been provided by the authors. 


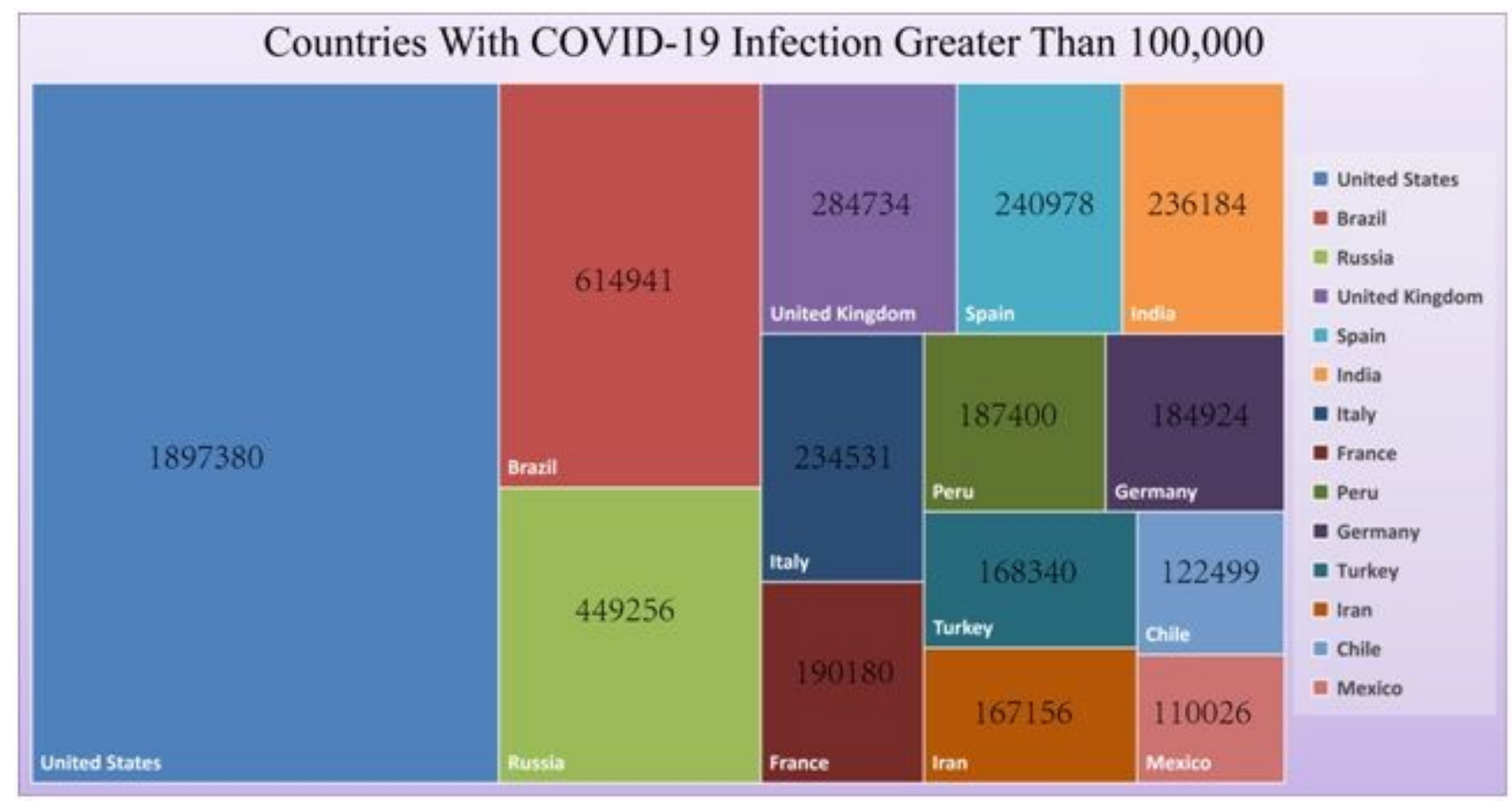

Figure 5

Countries With COVID-19 Infection Greater Than 100000 (a)

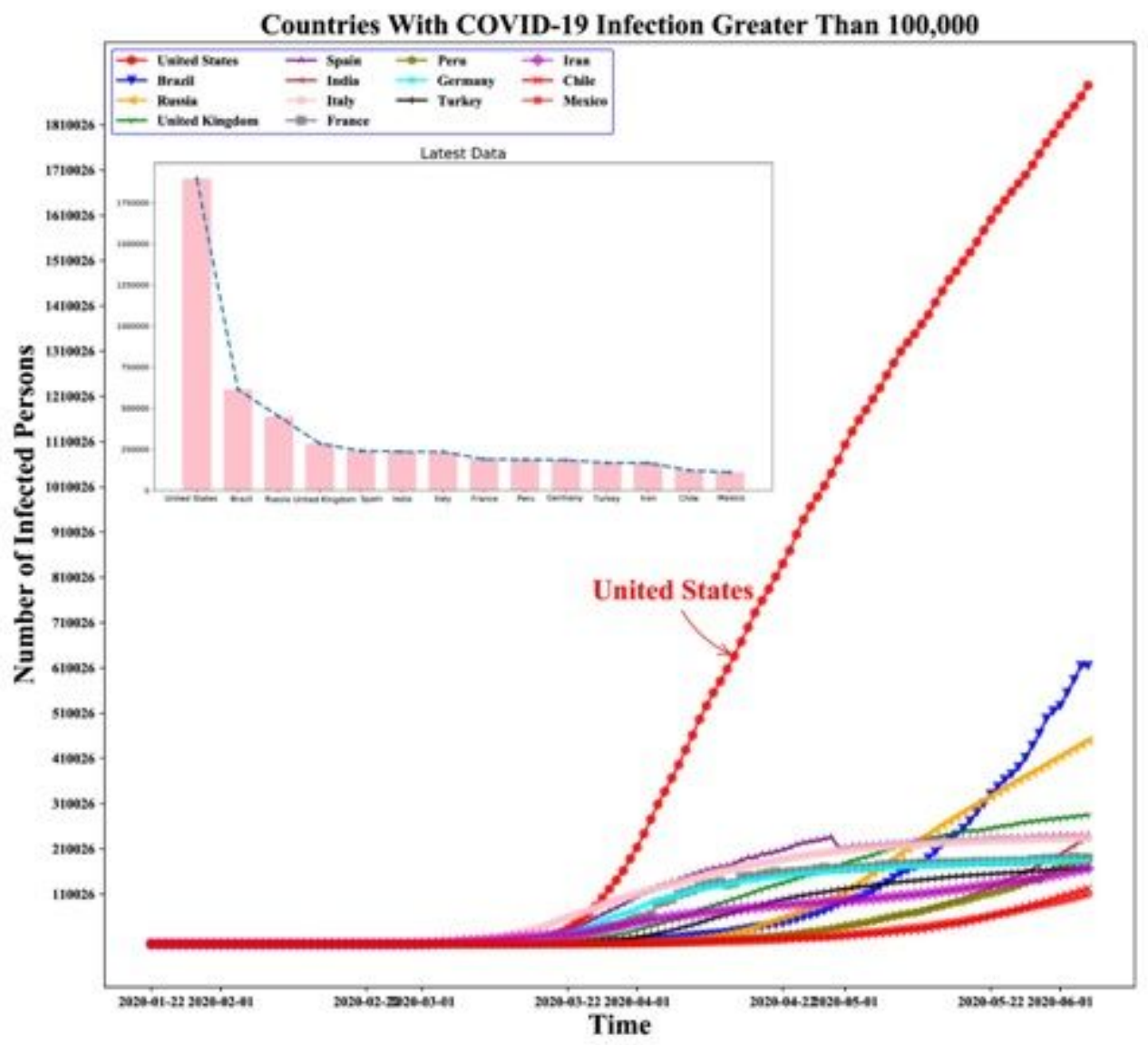

Figure 6 
Countries With COVID-19 Infection Greater Than 100000 (b)Note: The figure only analyzes countries with more than 100,000 people

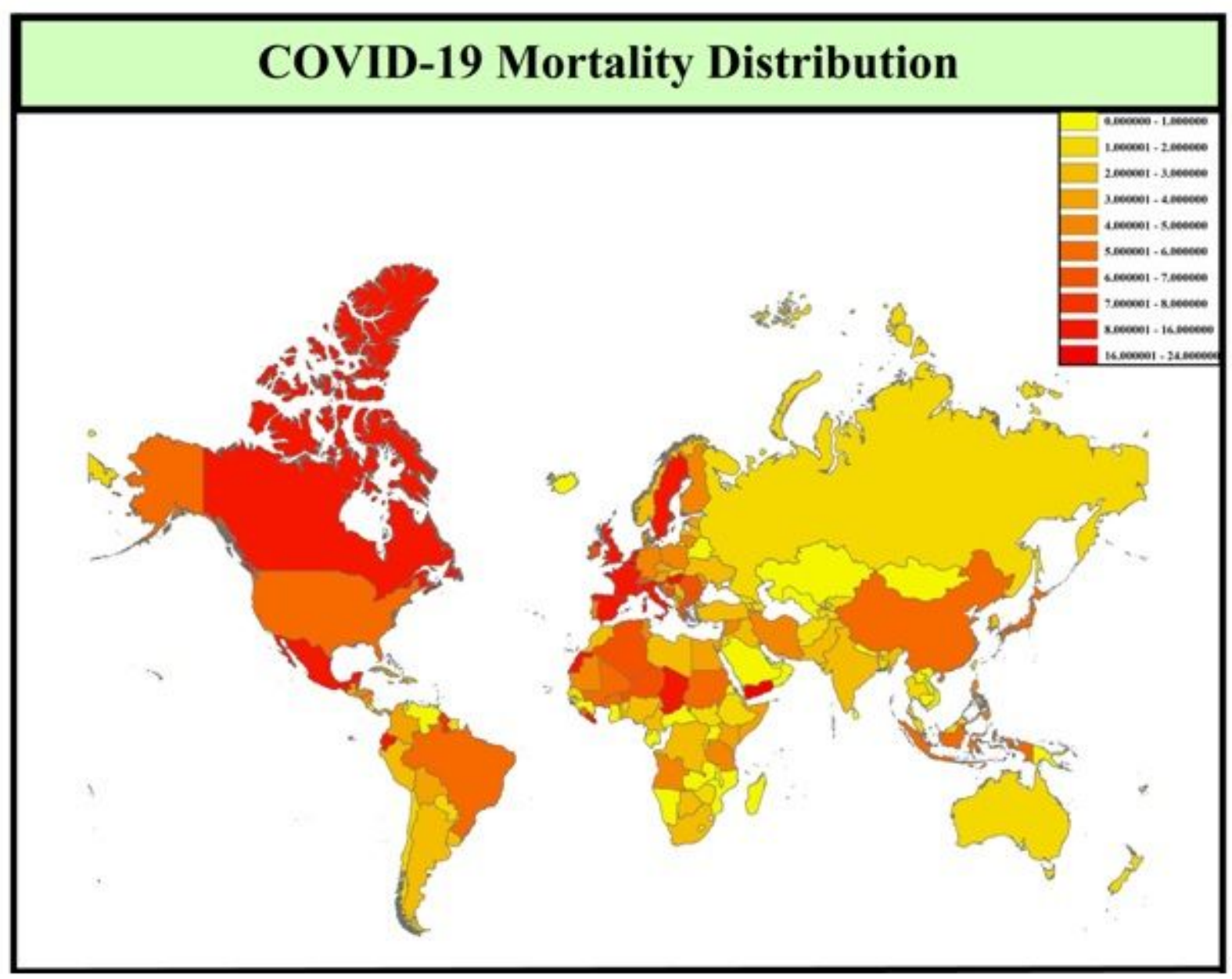

Figure 7

COVID-19 mortality distribution. Note: The designations employed and the presentation of the material on this map do not imply the expression of any opinion whatsoever on the part of Research Square concerning the legal status of any country, territory, city or area or of its authorities, or concerning the delimitation of its frontiers or boundaries. This map has been provided by the authors. 


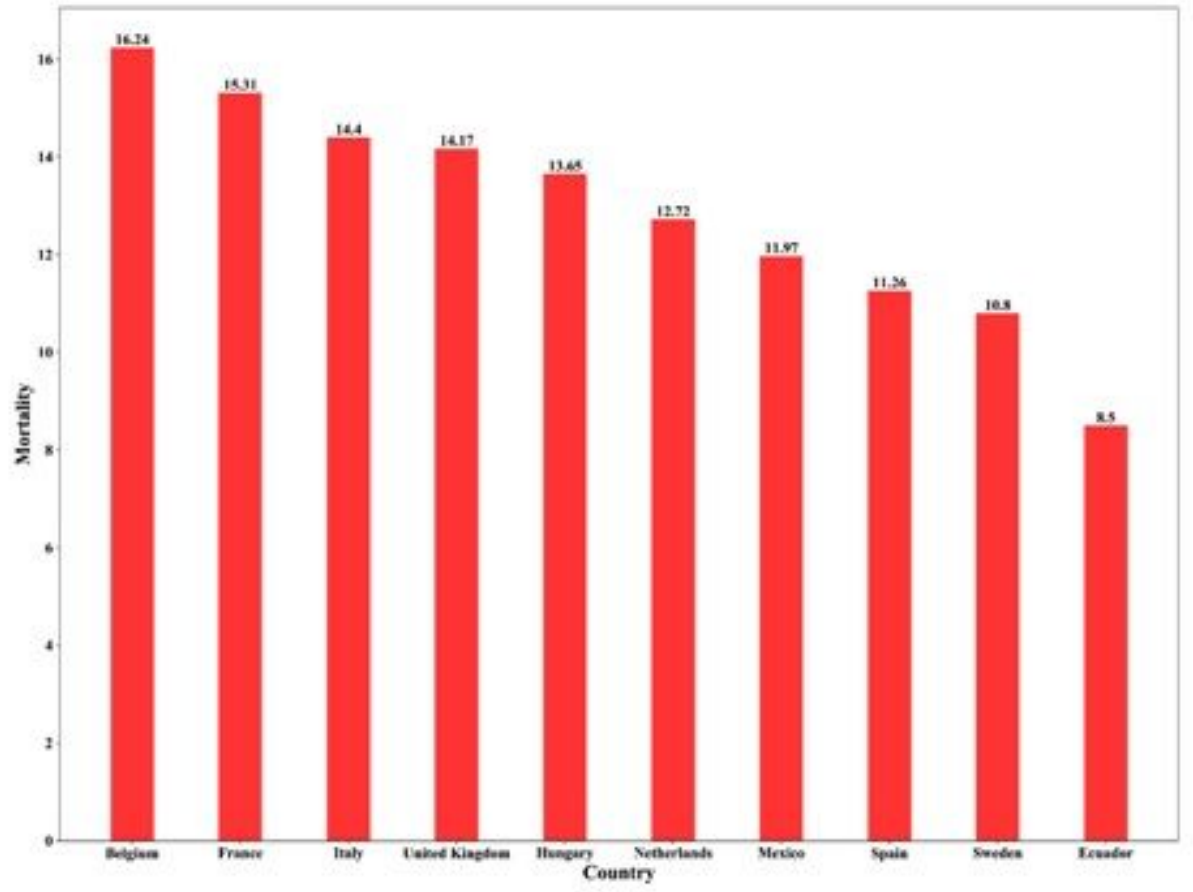

Figure 8

Top 10 Countries with COVID-19 Mortality

\section{COVID-19 Distribution in the United States}
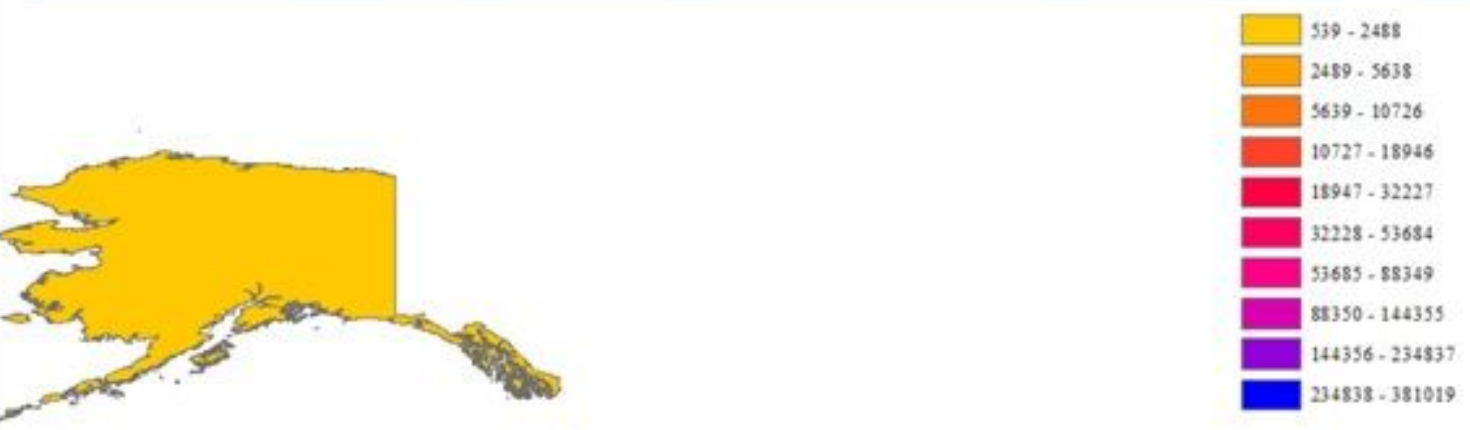


\section{Figure 9}

COVID-19 Distribution in the United States. Note: The red ellipse in the figure is the direction ellipse, Note: The designations employed and the presentation of the material on this map do not imply the expression of any opinion whatsoever on the part of Research Square concerning the legal status of any country, territory, city or area or of its authorities, or concerning the delimitation of its frontiers or boundaries. This map has been provided by the authors.

Analysis of COVID-19 Hot Spots in Major Regions of the United States

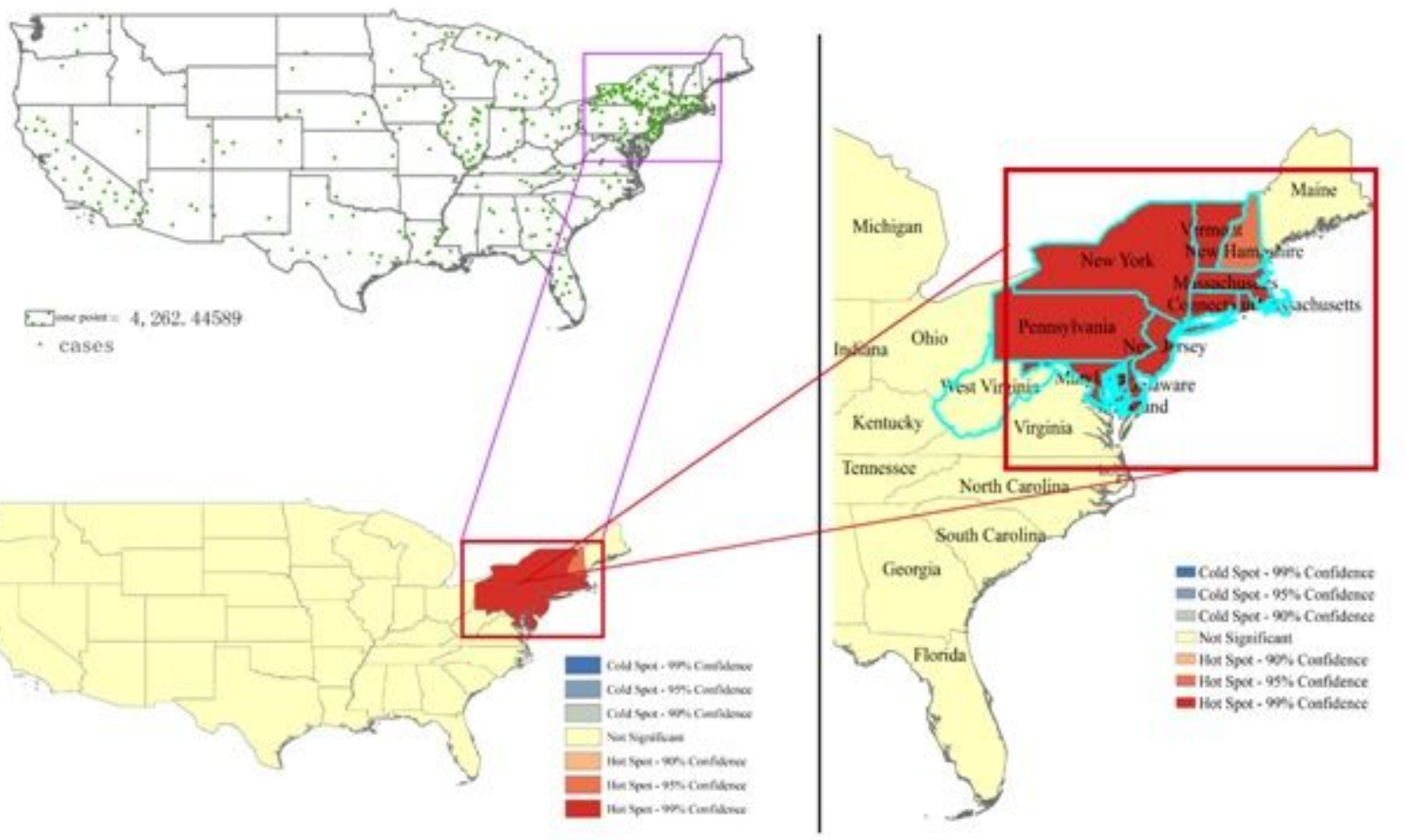

\section{Figure 10}

Analysis of COVID-19 Hot Spots in Major Regions of the United States 


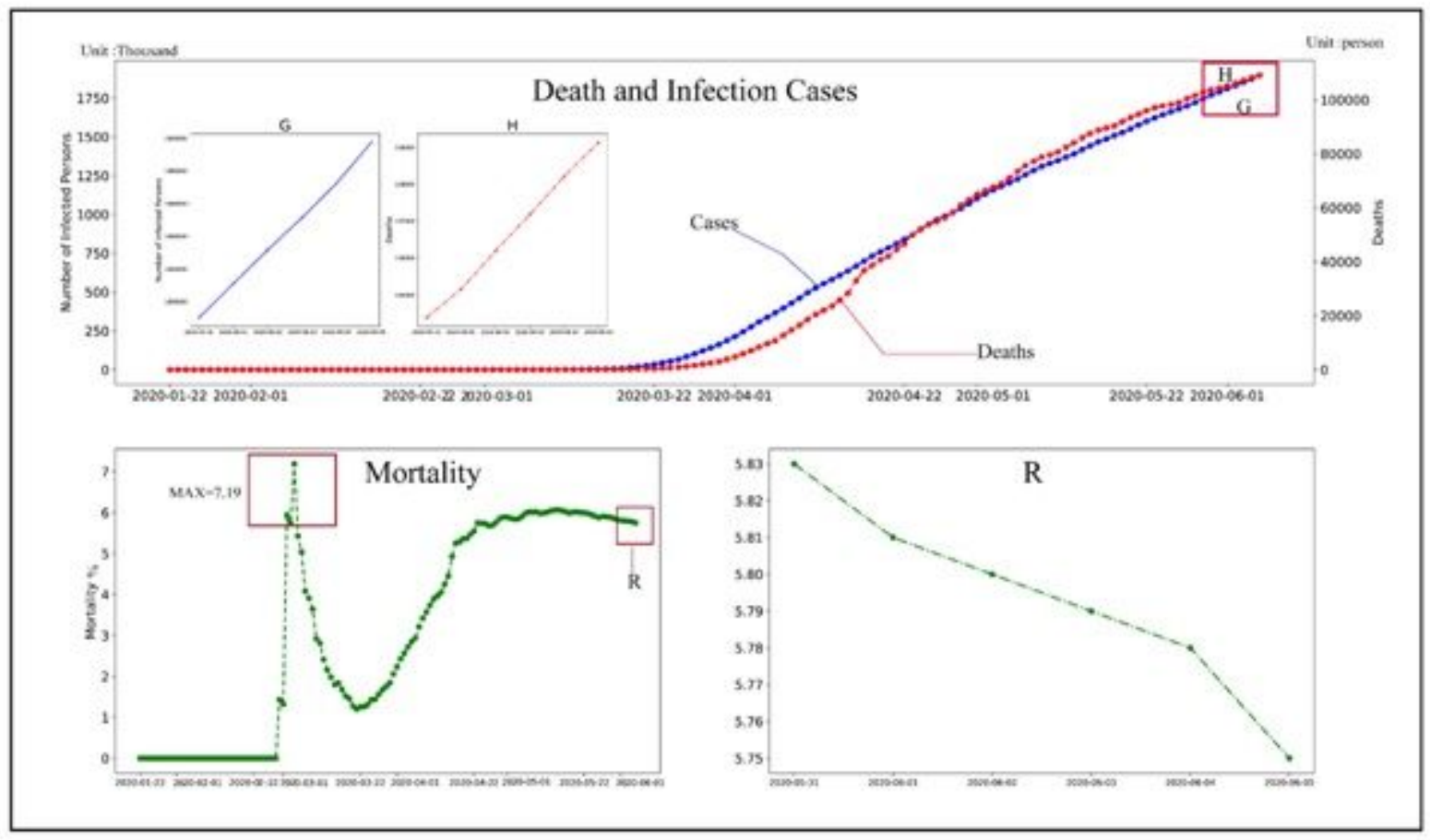

Figure 11

Time series visualization of infection cases, number of deaths and mortality in the United States.R is the latest mortality rate; $\mathrm{G}$ is the latest number of cases; $\mathrm{H}$ is the latest number of deaths. 